\title{
Learning-Induced Plasticity in Auditory Spatial Representations Revealed by Electrical Neuroimaging
}

\author{
Lucas Spierer, ${ }^{1}$ Eric Tardif, ${ }^{1}$ Holger Sperdin, ${ }^{1}$ Micah M. Murray,,${ }^{1,2,3}$ and Stephanie Clarke ${ }^{1}$ \\ ${ }^{1}$ Neuropsychology and Neurorehabiliation Service and ${ }^{2}$ Radiology Service, The Functional Electrical Neuroimaging Laboratory, Vaudois University \\ Hospital Center, and ${ }^{3} \mathrm{EEG}$ Core, Center for Biomedical Imaging of Lausanne and Geneva, Lausanne, 1011 Switzerland
}

Auditory spatial representations are likely encoded at a population level within human auditory cortices. We investigated learninginduced plasticity of spatial discrimination in healthy subjects using auditory-evoked potentials (AEPs) and electrical neuroimaging analyses. Stimuli were $100 \mathrm{~ms}$ white-noise bursts lateralized with varying interaural time differences. In three experiments, plasticity was induced with $40 \mathrm{~min}$ of discrimination training. During training, accuracy significantly improved from near-chance levels to $\sim 75 \%$. Before and after training, AEPs were recorded to stimuli presented passively with a more medial sound lateralization outnumbering a more lateral one (7:1). In experiment 1, the same lateralizations were used for training and AEP sessions. Significant AEP modulations to the different lateralizations were evident only after training, indicative of a learning-induced mismatch negativity (MMN). More precisely, this MMN at 195-250 ms after stimulus onset followed from differences in the AEP topography to each stimulus position, indicative of changes in the underlying brain network. In experiment 2, mirror-symmetric locations were used for training and AEP sessions; no training-related AEP modulations or MMN were observed. In experiment 3, the discrimination of trained plus equidistant untrained separations was tested psychophysically before and $0,6,24$, and $48 \mathrm{~h}$ after training. Learning-induced plasticity lasted $<6 \mathrm{~h}$, did not generalize to untrained lateralizations, and was not the simple result of strengthening the representation of the trained lateralizations. Thus, learning-induced plasticity of auditory spatial discrimination relies on spatial comparisons, rather than a spatial anchor or a general comparator. Furthermore, cortical auditory representations of space are dynamic and subject to rapid reorganization.

Key words: electroencephalography (EEG); auditory-evoked potential (AEP); electrical brain imaging; spatial; interaural time difference (ITD); training; plasticity; sound localization; mismatch negativity (MMN)

\section{Introduction}

Cerebral plasticity can occur within short delays after injury, sensory deprivation, or learning. Sensory representations can quickly reorganize after peripheral denervation of touch (Calford and Tweedale, 1988; Donoghue et al., 1990; Gerraghty and Kaas, 1991; Doetsch et al., 1996; Dinse et al., 1997; Faggin et al., 1997; Huntley, 1997; Barbay et al., 1999) or vision (Gilbert and Wiesel, 1992; Sur and Leamey, 2001; Calford et al., 2003). Striking effects on neural responses have also been observed in healthy animals after training with tools (Iriki et al., 1996; Iwamura, 2000) or specific auditory pitches (Edeline et al., 1993). Others have further demonstrated that modifications of receptive field properties were correlated with behavioral performance (Fritz et al., 2003, 2005; King, 2006).

Electrophysiological studies in humans have reported similar learning-induced plasticity. Näätänen et al. (1993) recorded

\footnotetext{
Received Nov. 1, 2006; revised March 20, 2007; accepted April 16, 2007.

This work was supported by The Swiss National Science Foundation (Grant 3100A0-103895/1 to S.C.). Cartool software (http://brainmapping.unige.ch/Cartool.htm) has been programmed by Denis Brunet from the Functional Brain Mapping Laboratory (Geneva, Switzerland) and is supported by the Center for Biomedical Imaging of Geneva and Lausanne. We thank Dr. Christoph Michel for providing additional analysis tools.

Correspondence should be addressed to either of the following: Lucas Spierer or Dr. Stephanie Clarke, Division Autonome de Neuropsychologie, Hôpital Nestlé, 5 Avenue Pierre Decker, Centre Hospitalier Universitaire Vaudois, 1011 Lausanne, Switzerland. E-mail: Lucas.spierer@chuv.ch or stephanie.clarke@chuv.ch.

DOI:10.1523/JNEUROSCI.0764-07.2007

Copyright $\odot 2007$ Society for Neuroscience $\quad$ 0270-6474/07/275474-10\$15.00/0
}

auditory-evoked potentials (AEPs) during the time that and after subjects were trained to discriminate the pitches of two complex stimuli. AEP modulations, measured as a mismatch negativity $(\mathrm{MMN})$, were only observed in subjects whose performance improved with training, whereas no changes were observed in subjects already performing well above chance levels before training. These results suggest that AEPs can be used as an index of training-induced neurophysiological changes and may furthermore be directly related to performance improvement. This hypothesis was further investigated by Gottselig et al. (2004), who recorded AEPs to tone sequences varying in the pitch of a single tone. Subjects actively discriminated tone sequences during a 6 min training session.

To date, studies investigating auditory plastic changes have focused on spectrally differing stimuli, including speech (Näätänen et al., 1993; Tremblay et al., 1997, 1998; Kraus et al., 1995; Menning et al., 2000; Atienza et al., 2002, 2005; Gottselig et al., 2004). Given the mounting evidence in humans for partially segregated brain networks mediating sound recognition and localization functions (Rauschecker, 1998; Hackett et al., 1999; Tian et al., 2001; Clarke and Thiran, 2004; De Santis et al., 2007), the present study addressed whether comparable plasticity occurs after learning to discriminate spatial features of otherwise identical acoustic stimuli. Psychophysical studies have shown that subjects can rapidly improve their ability to discriminate between 

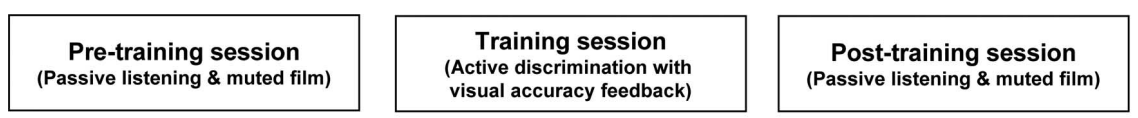

a. Experiment 1
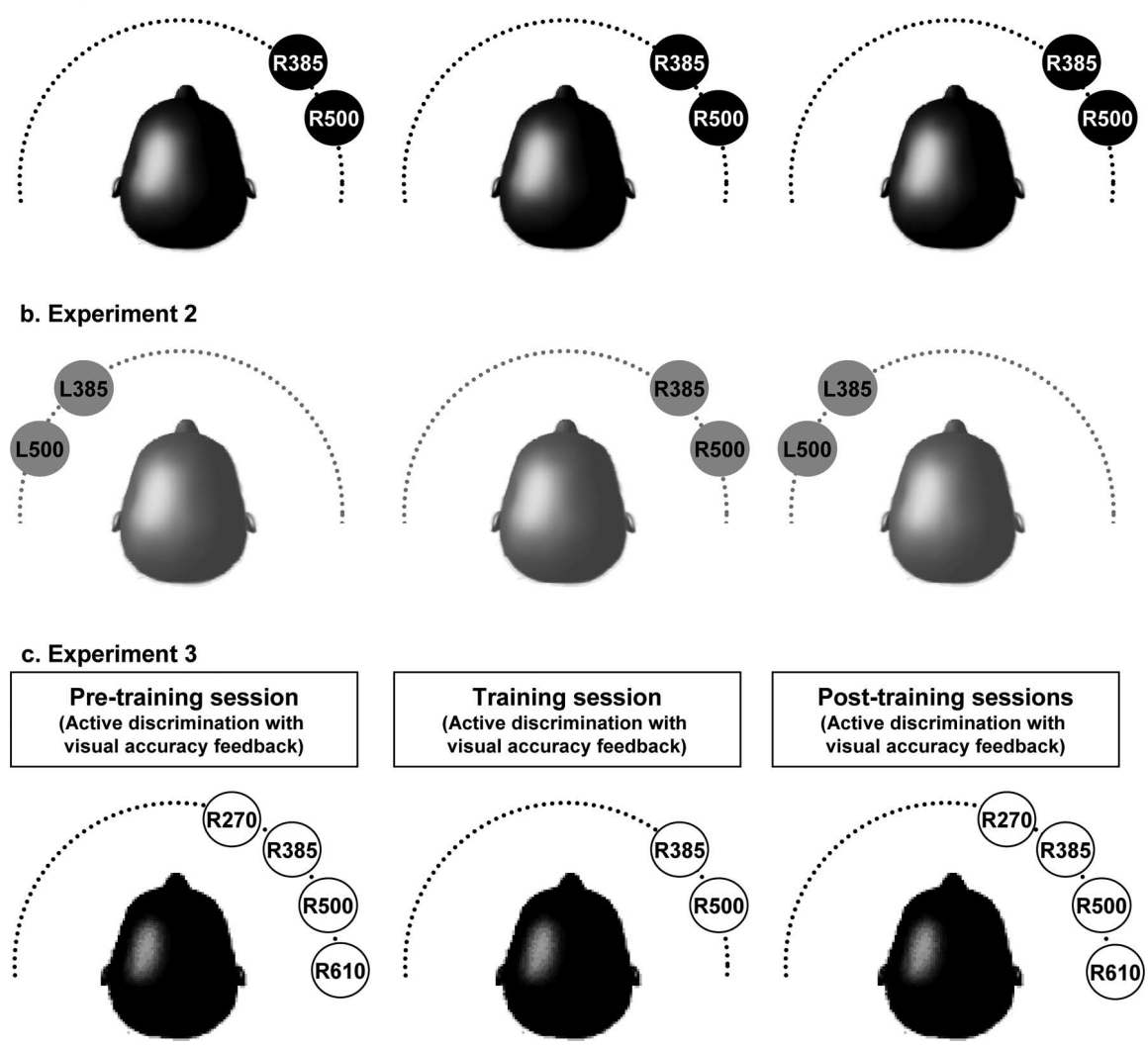

Figure 1. Experimental paradigm. Experiments 1 and 2 entailed three sessions, such that training, which involved active discrimination, was preceded and followed by passive listening sessions. $\boldsymbol{a}, \boldsymbol{b}$, The procedures for experiments 1 and 2 differed only in the loci of sound presentations during the pretraining $(\boldsymbol{a})$ and post-training $(\boldsymbol{b})$ sessions. For experiment 1 , these sounds were presented to the right hemispace (i.e., the same locias during the training session). For experiment 2, these sounds were presented to the left hemispace (i.e., the mirror-symmetric loci as during the training session). c, Experiment 3 entailed the same training session as experiments 1 and 2, as well as one pretraining and four post-training sessions (immediately after the training, 6, 24, and $48 \mathrm{~h}$ later). Pretraining and post-training sessions required active discrimination between pairs involving nearby positions.

sounds differing by small interaural time differences (ITDs) or interaural intensity differences (IIDs); two binaural cues for sound localization (Wright and Fitzgerald, 2001). Electrophysiological studies, most using MMN as their dependent measure, have shown that spatial information, like other acoustic features, is processed preattentively (Paavilainen et al., 1989; Schröger and Wolff, 1996; Nager et al., 2003; Altman et al., 2004; Tardif et al., 2006). We combined psychophysical and electrophysiological methods to investigate mechanisms mediating training-induced plasticity in auditory spatial representations. Three experiments investigated (1) the relationship between performance improvements and post-training electrophysiological measures, (2) whether training-induced plasticity in auditory spatial representations extends to untrained locations, and (3) whether it is the spatial representations themselves or rather mechanisms for their comparison that are modified through training.

\section{Materials and Methods \\ Subjects}

Twenty-six healthy volunteers participated and were divided in three age-matched groups, each of which completed one of the three experiments. Experiment 1 included 10 subjects (three females, two lefthanded using the Edinburgh questionnaire) (Oldfield, 1971) aged 21-28 years (mean age $\pm S D, 24.6 \pm 2.7)$. Experiment 2 included 10 subjects (four females, one lefthanded) aged $19-30$ years $(25.5 \pm 3.3)$. Experiment 3 included six subjects (five females, one left-handed) aged 24-27 years (25.7 \pm 0.5$)$. Each subject provided written, informed consent to participate in the study. No subject had a history of neurological or psychiatric illness. All reported normal hearing. All procedures were approved by the Ethics Committee of the Faculty of Biology and Medicine of the University of Lausanne. Each subject completed three sessions that we refer to as pretraining, training, and post-training (Fig. 1), which are detailed below.

\section{Stimuli}

Auditory stimuli were $100 \mathrm{~ms}$ white-noise bursts $(10 \mathrm{~ms}$ rise/fall envelope to minimize clicks; $44100 \mathrm{~Hz}$ digitization) generated using Adobe Audition 1.0 (Adobe Audition 1.0; Adobe Systems, San Jose, CA). Stimuli were lateralized by means of ITDs. Experiment 1 included two stimuli: with an ITD (right ear leading in time) of either 385 or $500 \mu$ s (hereafter termed R385 and R500, respectively). These sounds were lateralized to the right hemispace (see below). Experiment 2 included four stimuli. In addition to R385 and R500, which were used exclusively during the training session, mirror-symmetric lateralizations within the left hemispace were used exclusively during the pretraining and post-training sessions, corresponding to ITDs of -385 and $-500 \mu$ s (hereafter termed L385 and L500). This was achieved by reversing the headphones. ITDs of 385 and $500 \mu$ s led to perceived lateralizations $\sim 40^{\circ}$ and $50^{\circ}$ from the central midline, respectively [according to Blauert's complex formula (Blauert, 1997)]. Experiment 3 included four stimuli: R385, R500, and two additional positions with an ITD of either 270 or $615 \mu$ s (hereafter termed R270 and R615, respectively), which led to perceived lateralizations $\sim 30^{\circ}$ and $60^{\circ}$ from the central midline, respectively. Each sound was presented via insert earphones (model ER-4P; Etymotic Research, Elk Grove Village, IL) at $86 \pm 3 \mathrm{~dB}$ (measured using a CESVA SC-L sound pressure meter; CESVA Instruments, Barcelona, Spain).

\section{Procedure and task}

The rationale for the three experiments was the following. To determine whether spatial training in one hemispace generalized to the other hemispace, subjects of experiments 1 and 2 underwent the same training within the right hemispace, and effects of training on spatial discrimination were then assessed either at trained (experiment 1) or untrained (experiment 2) lateralizations. This design allowed us to ensure both groups showed behavioral improvement for the same spatial locations and also to control for the possibility of general repetition effects intertwined with effects of training-induced plasticity. Both behavioral and EEG data were collected throughout the length of experiments 1 and 2. Experiment 3 was conducted to better determine the basis of any training-related effects observed during experiments 1 and 2. Specific stimulus details are provided below. Briefly, we included additional pairs of adjacent lateralizations to disentangle whether subjects were learning spatial discrimination specific to the trained locations (R385 and R500) or more generally the discrimination of a relative spatial separation between sounds regardless of their specific lateralization. These pairings also allowed us to assess whether training-induced effects followed from subjects specifically learning and forming a spatial "template" or "anchor" of the R385 
position; in which case, performance enhancement for all pairs involving the R385 position would be predicted. Second, we included sound pairs wherein the R500 lateralization was presented first (i.e., in contrast to the order used during the training session) to assess whether traininginduced enhancement was sequence specific. Finally, participants in experiment 3 were also tested at multiple delays after training to assess the persistence of training-induced performance enhancements. Stimulus delivery and response recording were controlled by E-Prime (Psychology Software Tools, Pittsburgh, PA) while subjects sat in an electrically shielded and sound-attenuated room.

\section{Pretraining and post-training sessions}

In experiment 1, pretraining and post-training sessions consisted of pseudo-randomized presentations of R385 and R500 stimuli (Fig. 1a), which were respectively presented 87.5 and $12.5 \%$ of the time with the constraint that at least one R385 stimulus always preceded an R500 stimulus. Stimuli were blocked into series of 360 trials with an interstimulus interval of $1 \mathrm{~s}$. Each participant completed five blocks before and five after the training session while watching a muted film with subtitles in their native language. They were given no instructions about the auditory stimuli, except to ignore them and to watch the film. Experiment 2 followed an identical procedure to that of experiment 1, except that L385 and L500 were presented instead of R385 and R500 (Fig. 1b).

Participants in experiment 3 underwent the same training session as used in experiments 1 and 2. Pretraining and post-training sessions were completed as follows: just before $(\mathrm{H}-0)$, just after $(\mathrm{H}+0), 6 \mathrm{~h}$ after $(\mathrm{H}$ $+6), 24 \mathrm{~h}$ after $(\mathrm{H}+24)$, and $48 \mathrm{~h}$ after $(\mathrm{H}+48)$ the training session. These sessions also consisted of a two-alternative forced-choice task. Stimuli duration, interstimulus and intertrial interval, response window, and visual feedback were the same as during the training session. Subjects discriminated whether the two items of the following stimulus pairs were the same or different: R270-R270, R270-R385, R385-R385, R385-R500, R500-R385, R500-R500, R500-R610, and R610-R610. Stimuli were blocked into series of 192 trials $(24 \times 8$ pairs), subjects underwent two blocks per session. The procedure and calculation of sensitivity were the same as in the training session (see below).

\section{Training session}

In experiments 1,2 , and 3 , the training session consisted of a twoalternative forced-choice task. Subjects discriminated whether the two items of stimulus pairs R385-R385 or R385-R500 were the same or different. Subjects pressed the left response-box button when they perceived the two stimuli of a pair at the same location and the right button if the stimuli were perceived at different locations. Stimuli of each pair were separated by $250 \mathrm{~ms}$ (from offset to onset) and were blocked into a series of 200 trials. Subjects were required to respond within $750 \mathrm{~ms}$ after trial offset. A fixed $1000 \mathrm{~ms}$ intertrial interval (ITI) followed response initiation (or $750 \mathrm{~ms}$ after trial offset in the case of an absent response). Visual feedback was given following responses, indicating whether the response was correct (green square), incorrect (red square), or too slow (yellow square). Participants completed five training blocks in a session that lasted $\sim 40 \mathrm{~min}$. Behavioral data for each block were analyzed according to signal detection theory (Green and Swets, 1966). Sensitivity $\left(d^{\prime}\right)$ was calculated according to the following equation: $d^{\prime}=z(\mathrm{H})-z(\mathrm{FA})$, where $z(\mathrm{H})$ and $z(\mathrm{FA})$ represent the transformation of the hit and falsealarm rates into $z$ scores (Macmillan and Creelman, 1991). In experiments 1 and 2, hits were the R385-R500 trials, reported as originating from different locations, and false alarms were R385-R385 trials, reported as originating from different locations.

\section{EEG acquisition and preprocessing}

Continuous EEG was acquired at $512 \mathrm{~Hz}$ through a 128 channel Biosemi ActiveTwo system (Biosemi, Amsterdam, Netherlands) referenced to the CMS-DRL ground (which functions as a feedback loop driving the average potential across the montage as close as possible to the amplifier zero). Details of this circuitry, including a diagram can be found on the Biosemi website (http://www.biosemi.com/pics/zero_ref1_big.gif). All analyses were conducted using CarTool software (http://brainmapping.unige.ch/Cartool.htm). Epochs of EEG from $98 \mathrm{~ms}$ prestimulus to 486 ms poststimulus onset (i.e., 50 data points before and 250 data points after stimulus onset) were averaged for each of the stimulus position and from each subject. To maintain equivalent signal-to-noise ratios for AEPs in response to each condition, all trials in response to infrequent stimuli (i.e., in experiment 1, R500; in experiment 2, L500) and only trials from frequent stimuli (i.e., in experiment 1, R385; in experiment 2, L385) that immediately preceded infrequent trials were considered during epoching. In addition to the application of an automated artifact criterion of $\pm 100 \mu \mathrm{V}$, epochs with blinks, eye movements, or other sources of transient noise were also rejected. Baseline correction was applied to the $98 \mathrm{~ms}$ prestimulus period. Before group averaging, data at artifact electrodes from each subject were interpolated (Perrin et al., 1987), and the data were recalculated against the average reference and bandpass filtered $(0.68-40 \mathrm{~Hz})$.

\section{EEG analyses and source estimation}

General analysis strategy. The main objective of this study was to examine the spatiotemporal mechanisms mediating plasticity in the spatial representation of sounds. Electrophysiological analyses, based on an electrical neuroimaging approach, using both local and global measures of the electric field at the scalp, were used. These allowed us to differentiate effects following from modulation in the strength of responses of statistically indistinguishable brain generators from alterations in the configuration of these generators (viz. the topography of the electric field at the scalp) as well as latency shifts in brain processes across experimental conditions. Because the electrophysiological methods have been extensively detailed previously (Michel et al., 2004; Murray et al., 2004; De Santis et al., 2007; for a recent publication of formulas, see Murray et al., 2006), we provide only the essential details here.

AEP waveform analyses. As a first level of analysis and to minimize the possibility of missed effects (type II errors), we analyzed waveform data from all electrodes as a function of time after stimulus onset in a series of pairwise comparisons ( $t$ tests). Correction was made for temporal autocorrelation at individual electrodes (Guthrie and Buchwald, 1991) through the application of an 11 contiguous data-point temporal criterion for the persistence of differential effects. The results of this analysis are presented as an intensity plot with the $x$-, $y$-, and $z$-axes, respectively, representing time (after stimulus onset), electrode location, and the $t$ test result (indicated by a color value) at each data point. We would emphasize that although these analyses give a visual impression of specific effects within the dataset, our conclusions are principally based on global measures of the electric field at the scalp.

Topographic analyses. Topographic analyses were conducted in two ways. The first was a topographic pattern analysis on the collective dataset for each experiment (i.e., both as a function of time within each AEP and also a function of stimulus position and pretraining vs post-training sessions). This pattern analysis uses a hierarchical agglomerative clustering algorithm to identify the predominant topographies (i.e., maps) and their sequence within a given dataset (these methods are implemented in Cartool software) (see also Tibshirani et al., 2005). The optimal number of maps (i.e., the minimal number of maps that accounts for the greatest variance of the dataset) is determined using a modified Krzanowski-Lai criterion (Krzanowski and Lai, 1985).

Importantly, the topography of the electric field is independent of reference electrode (cf. Michel et al., 2004), and the pattern analysis is insensitive to pure amplitude modulations across conditions because normalized maps are compared. The pattern of maps observed in the group-averaged data were statistically tested by comparing each of these maps with the moment-by-moment scalp topography of individual subjects' AEPs from each condition. In other words, the methods described above generate a hypothesis concerning the sequence of maps observed in the AEPs and any differences between stimulus positions and/or sessions. To statistically assess this hypothesis, each time point of each AEP from each subject was labeled according to the map with which it best correlated spatially (Brandeis et al., 1995; Murray et al., 2006). We, hereafter, refer to this procedure as "fitting." This fitting method determines whether a given stimulus lateralization and/or experimental session is more often described by one map versus another, and therefore if different generator configurations better account for particular conditions/ sessions. In addition to testing for modulations in the electric field to- 
pography across conditions, this analysis also provides a more objective means of defining AEP components. That is, we here define an AEP component as a time period of stable scalp topography.

The second analysis of topography entailed calculation of the global dissimilarity between two maps (Lehmann and Skrandies, 1980). Global dissimilarity is an index of configuration differences between two electric fields that is independent of their strength (normalized data are compared). Values can range from 0 to 2, with 0 indicative of topographic homogeneity and 2 indicative of topographic inversion. It is calculated as the root mean square of the difference between two normalized maps and is statistically assessed using a Monte Carlo nonparametric bootstrapping procedure (Manly, 1991). This analysis is currently limited to pairwise contrasts. Thus, in the present study, dissimilarity was always measured between AEPs to the two lateralized stimuli from the pretraining and post-training sessions, separately. Because electric field changes are indicative of changes in the underlying generator configuration (Lehmann, 1987), this test provides a statistical means of determining if and when the brain network activated by 385 and $500 \mu$ sTD cues differ.

Source estimations. We estimated the sources in the brain underlying the AEPs from each stimulus type and sessions using the local autoregressive averages (LAURA) distributed linear inverse solution (Grave de Peralta Menendez et al., 2001; 2004; for a comparison of inverse solution methods, see Michel et al., 2004). LAURA selects the source configuration that better mimics the biophysical behavior of electric vector fields (i.e., activity at one point depends on the activity at neighboring points according to electromagnetic laws). The solution space was calculated on a realistic head model that included 4024 nodes, selected from a $6 \times 6 \times$ $6 \mathrm{~mm}$ grid equally distributed within the gray matter of the average brain of the Montreal Neurological Institute. The results of the above topographic pattern analysis defined time periods for which intracranial sources were estimated. We emphasize that these estimations, in the present study, provide visualization, rather than a statistical analysis, of the likely underlying sources.

\section{Results}

\section{Behavioral results}

Training improved performance on auditory spatial discrimination during each of the three experiments (Fig. 2a). During experiment 1 , all but one subject had a $d^{\prime}$ value $<1.0$ on the first training block, indicating that discriminating R385 and R500 lateralizations was difficult. Performance accuracy on the first block was $62.3 \pm 3.9 \%$ correct (mean \pm SEM) and on the fifth block was $79.3 \pm 3.9 \%$ correct. Performance improved significantly across training blocks ( $d^{\prime}$ submitted to a one-way repeatedmeasure ANOVA showed a main effect of block; $F_{(1,9)}=13.12$; $p<0.01)$. Performance was significantly better on the fifth block than on either the first $\left(t_{(9)}=4.681 ; p<0.001\right)$ or the second blocks $\left(t_{(9)}=4.944 ; p<0.001\right)$. Performance was also better on the fourth than either the first $\left(t_{(9)}=4.048\right.$; $\left.p<0.003\right)$ or the second blocks $\left(t_{(9)}=3.862 ; p<0.005\right)$. During experiment 2 , all but three subjects had $d^{\prime}$ values $<1.0$ on the first block. Performance accuracy on the first block was $63.6 \pm 3.9 \%$ correct and on the fifth block was $72.2 \pm 4.0 \%$ correct. As in experiment 1 , submission of $d^{\prime}$ values to an ANOVA revealed that performance improved significantly across training blocks (main effect of block; $F_{(1,9)}=14.60 ; p<0.01$ ). Performance was significantly better on the fifth than the first block $\left(t_{(9)}=2.843 ; p<0.03\right)$ and in the fourth than for the first block $\left(t_{(9)}=2.876 ; p<0.03\right)$; this pattern of results mirrors that observed for experiment 1 . During experiment 3 , all but two subjects had $d^{\prime}$ values $<1.0$ on the first block. Performance accuracy on the first block was $65.2 \pm 7.7 \%$ correct and on the fifth block was $75 \pm 6.5 \%$ correct. As in experiments 1 and 2, submission of $d^{\prime}$ values to an ANOVA revealed that performance improved significantly across training blocks (main effect of block; $F_{(1,6)}=20.95 ; p<0.05$ ). Performance was significantly better on the fifth than the first block a. Training performance

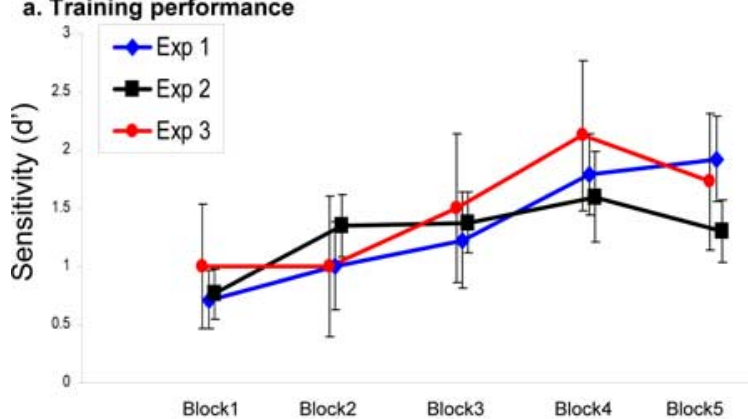

b. Discrimination performance in pre- and post-sessions of Experiment 3

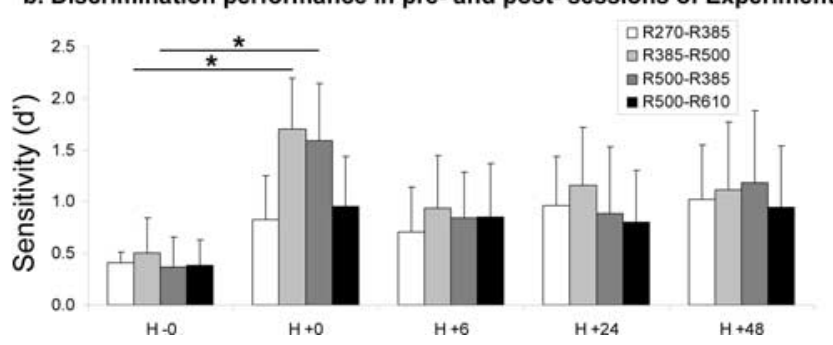

Figure 2. Behavioral results. $\boldsymbol{a}$, Group-averaged sensitivity ( $d^{\prime} \pm$ SEM) in discriminating sound lateralizations is plotted as a function of training block for experiment 1 (blue line), experiment 2 (black line), and experiment 3 (red line). Sensitivity significantly increased with training for both experiments, with no evidence of differences between participants in experiments 1, 2, and 3 (see Results for details). $\boldsymbol{b}$, Group-averaged sensitivity ( $d^{\prime} \pm$ SEM) in discriminating sound lateralizations is plotted as a function of pretraining and post-training sessions for experiment 3. The training improved discrimination performance only for pairs involving the two trained positions and did not persist over time. Exp, Experiment. ${ }^{*} p<0.05$.

$\left(t_{(5)}=6.60 ; p<0.003\right)$ and on the fourth than the first block $\left(t_{(5)}=7.74 ; p<0.01\right)$. This pattern of results was identical to that of experiments 1 and 2 . Sensitivity measures $\left(d^{\prime}\right)$ from all three experiments were submitted to a repeated-measures ANOVA using the experiment as a between subjects factor and the block as the within subjects factor. The main effect of block was significant $\left(F_{(1,26)}=3.319 ; p<0.03\right)$ and the main effect of group failed to meet the 0.05 significance criterion $\left(F_{(1,26)}=1.962 ; p>0.8\right)$. These results indicate similar performance improvement with training across all three experiments (Fig. 2a).

Inspection of $d^{\prime}$ values from experiment 3 suggests that training improved discrimination performance only during the session immediately after training (i.e., $\mathrm{H}+0$ and not $\mathrm{H}+6, \mathrm{H}+$ 24 , and $\mathrm{H}+48$ ) and only for pairs involving both of the trained positions. Training effects did not persist $6 \mathrm{~h}$ after training and did not generalize to adjacent untrained positions (Fig. $2 b$ ). Sensitivity $\left(d^{\prime}\right)$ values from the pretraining and post-training sessions were submitted to an ANOVA. There was a significant interaction between the within-subjects factors of stimulus pair and $\operatorname{session}\left(F_{(2,6)}=2.364 ; p<0.05\right)$. We therefore conducted additional ANOVAs that were limited to each stimulus pair. Significant main effects of session were observed only for pairs involving both of the trained positions (R385-R500: $F_{(1,6)}=8.42, p<$ 0.003; R500-R385: $\left.F_{(1,6)}=4.47 ; p<0.05\right)$, for all other pairs, the effect of session failed to meet the 0.05 significance criterion. Post hoc analyses revealed that sensitivity improved significantly between $\mathrm{H}-0$ and $\mathrm{H}+0$ for the R385-R500 and R500-R385 pairs $\left(t_{(5)}=6.73, p<0.003\right.$ and $t_{(5)}=3.89, p<0.03$, respectively). But this effect declined significantly within $6 \mathrm{~h}$ after the training [i.e., $\mathrm{H}+0$ vs $\mathrm{H}+6$ (R385-R500: $t_{(5)}=4.90, p<0.005$; R500-R385: $\left.t_{(5)}=3.17, p<0.03\right) ; \mathrm{H}+0$ vs $\mathrm{H}+24\left(\mathrm{R} 385-\mathrm{R} 500: t_{(5)}=2.72\right.$, 
$p<0.05$; R500-R385: $t_{(5)}=4.86, p<$ 0.005); $\mathrm{H}+0$ vs $\mathrm{H}+48$ (R385-R500: $t_{(5)}=3.24, p<0.03$; R500-R385: $t_{(5)}=$ 4.86, $p=0.055)]$. To test whether training-induced performance benefits already began to decline during the course of the $\mathrm{H}+0$ session itself, we split the data from this session in half chronologically and submitted them to a $2 \times 4$ ANOVA using session portion (first vs second half) and stimulus pair as within subjects factors. The main effect of session portion failed to meet the 0.05 significance criterion $\left(F_{(1,6)}=0.926 ; p=0.38\right)$, suggesting that sensitivity did not decline over the course of the $\mathrm{H}+0$ session.

Despite the short duration of plasticity $(<6 \mathrm{~h})$, such a procedure may be useful in a clinical setting for training-based rehabilitation. Indeed, previous studies have shown that repetitive application of treatment with transient effects may lead to long-lasting changes (Schindler et al., 2002).

\section{AEP waveform analyses}

Figure 3 shows group-averaged AEPs in response to each session, stimulus condition, and experiment from an exemplar frontal electrode. This figure also illustrates the results of paired contrasts across the entire electrode montage separated between pretraining and post-training sessions for experiments 1 and 2. These contrasts reveal that AEP differences between the two stimulus positions were observed only for experiment 1 during the posttraining session. Significant differences were widespread over the electrode montage during the $\sim 200-300$ ms poststimulus onset interval. No robust differences were observed in the pretraining session of either experiment. Nor were differences observed during the post-training session of experiment 2. Given this apparent selectivity of effects when the data from each electrode and session were separately analyzed, we focus our discussion on the analyses of the global electric field on the data from experiment 1, although all analyses were conducted on both experiments. As noted in Materials and Methods, analyses of the global electric field were prioritized because they are reference independent and also provide statistically based information on whether effects stem from topographic and/or strength modulations.

\section{Topographic pattern analysis and global} dissimilarity analysis

The output of the topographic pattern analysis of the collective data from experiment 1 is displayed in Figure 4a. Multiple maps were identified in the group-averaged data over the 0-39 ms interval, and these were differentially observed across sessions but not lateralizations. That is, there was a significant interaction between session and map over 0-39 ms after submitting these maps observed at the group-average level to the fitting procedure at the individual subject level $\left(F_{(1,9)}=7.19 ; p=0.025\right)$. Addi- tional ANOVAs were therefore conducted for each session, separately. Although both maps were equally often observed during the pretraining session (i.e., no main effects or interactions were observed), the map framed by a dotted line was significantly more often observed during the post-training session, as indicated by the main effect of the map $\left(F_{(1,9)}=10.24 ; p=0.011\right)$. Single maps were identified in common across both lateralizations and both sessions over the 41-72, 74-135, 137-193, and 301-488 ms periods.

In contrast, over the 195-250 ms poststimulus period, different maps described AEPs in response to the R500 and R385 conditions during the post-training but not pretraining session. These maps are framed in black and dark green, respectively, in Figure $4 a$. The global dissimilarity between these maps is 0.374 . As above, this pattern observed in the group-averaged data were tested in the data of individual subjects, using the abovementioned fitting procedure (see Materials and Methods). The values of the fitting procedure (i.e., the amount of time over the 195-250 ms interval when each of the framed maps in Fig. $4 a$ better correlated spatially with the data from each subject and condition, hereafter termed "map presence") were then submitted to a repeated-measures ANOVA using session, stimulus lateralization, and map as within-subject factors (Fig. 4b). There 


\section{a. Topographic pattern analysis}

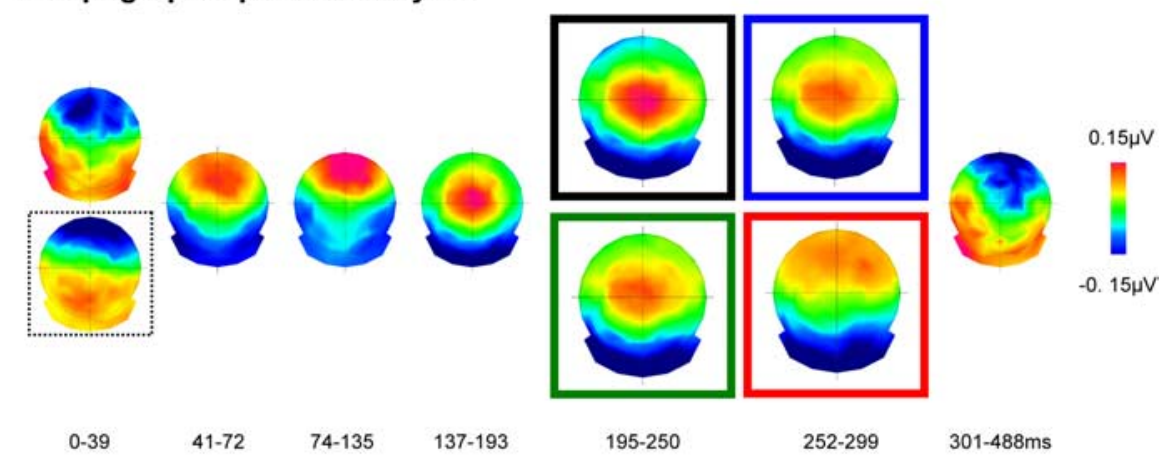

b. Fitting procedure (195-250ms interval)

d. Predictive value of initial training

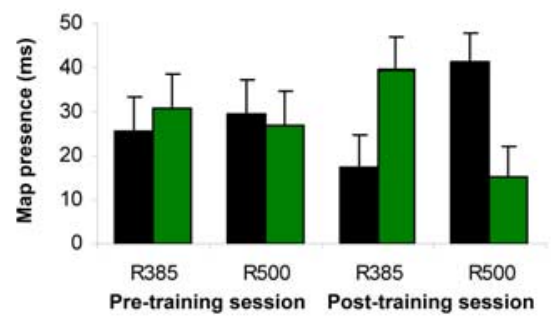

c. Fitting procedure $(252-299 \mathrm{~ms}$ interval)
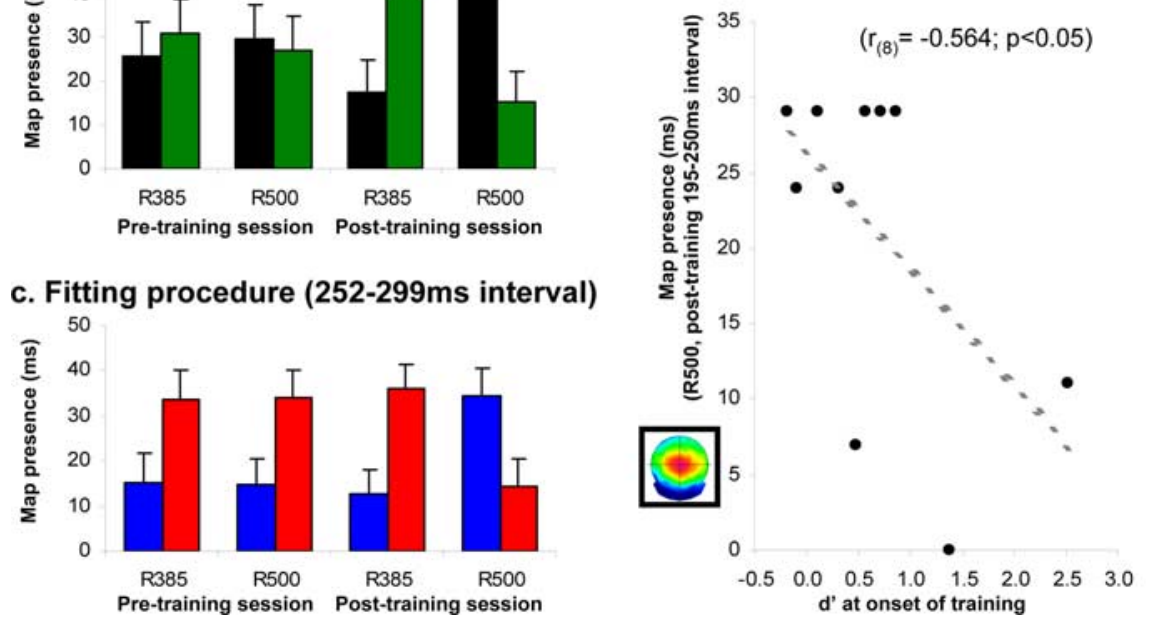

Figure 4. Electrical neuroimaging results. $\boldsymbol{a}$, The topographic pattern analyses identified seven time periods of stable topography across the collective $488 \mathrm{~ms}$ poststimulus period from the four conditions of experiment 1 . All topographies are shown with the nasion upward and left scalp leftward. For some of these time periods, multiple topographies were identified in the groupaverage AEPs. These topographies are framed. The reliability of this observation at the group-average level was then assessed at the single-subject level using the fitting procedure (see Materials and Methods). $\boldsymbol{b}$, Over the 195-250 ms poststimulus period, different maps (framed in black and dark green) described AEPs in response to the R500 and R385 conditions during the posttraining but not pretraining session. There was a significant three-way interaction between session, stimulus lateralization, and map. Additional analyses further revealed a significant two-way interaction between stimulus lateralization and map. Error bars indicate SEM. c, Over the 252-299 ms poststimulus period, different maps again described AEPs in response to the R500 and R385 conditions during the post-training but not pretraining session (framed in gray and light green). As above, there was a significant three-way interaction between session, stimulus lateralization, and map. Additional analyses further revealed a significant twoway interaction between stimulus lateralization and map. Error bars indicate SEM. $\boldsymbol{d}$, The presence of maps in response to R500 after training significantly correlates with the initial discrimination performance $\left(d^{\prime} 1\right)\left(r_{(9)}=0.567 ; p<0.05\right)$.

different configurations of intracranial generators (Lehmann, 1987), this pattern of results indicates that training lead to the activity of (partially) distinct brain networks in response to each of the two stimulus positions. We also tested for a linear correlation between map presence over the $195-250 \mathrm{~ms}$ interval and sensitivity at the onset of training to assess whether performance measures could be used to predict electrophysiological plasticity, which would be particularly important for planned extension of this research to clinical populations. We observed a significant negative correlation between sensitivity at the onset of training and the presence of the "black" map post-training $\left(r_{(8)}=-0.564 ; p<0.05\right)$, suggesting that the more a subject has room to improve his/her performance, the more the black map will account for his/her post-training AEPs in response to the R500 stimulus (Fig. 4d). We would note that this analysis does not account for how training directly impacted a subject's performance. Rather, this analysis would suggest that pretraining performance is predictive of posttraining AEP topography, and by extension, configurations of intracranial generators.

Over the 252-299 ms poststimulus period, different maps described AEPs in response to the R500 and R385 conditions during the post-training but not pretraining session. These maps are framed in gray and light green, respectively, in Figure $4 a$. The global dissimilarity between these maps is 0.469 . After the fitting procedure, analysis of map presence over this period revealed significant interactions between session and map $\left(F_{(1,9)}=5.17 ; p=0.049\right)$ and between stimulus lateralization and $\operatorname{map}\left(F_{(1,9)}=8.15 ; p=0.019\right)$. Additional ANOVAs were therefore conducted for each session, separately. For the pretraining session, there was a main effect of map $\left(F_{(1,9)}=5.33 ; p=0.046\right)$. Neither the main effect of stimulus lateralization nor

was a significant interaction between session, stimulus lateralization, and map $\left(F_{(1,9)}=6.98 ; p<0.03\right)$ over the $195-250 \mathrm{~ms}$ poststimulus period. There was also a significant interaction between stimulus lateralization and map $\left(F_{(1,9)}=6.99 ; p<0.03\right)$. None of the other main effects (stimulus lateralization or map) or any other interaction reached our 0.05 significance criterion. We therefore conducted additional ANOVAs for each session, separately. For the pretraining session, neither main effect nor the interaction reached the 0.05 significance criterion. For the posttraining session, there was a significant interaction between stimulus lateralization and map $\left(F_{(1,9)}=8.39 ; p=0.018\right)$. That is, the map framed in black was more often observed in response to the R500 condition, whereas the map framed in dark green was more often observed in response to the R385 condition (Fig. $4 b$ ). Because different topographies at the scalp forcibly follow from the interaction between factors reached the 0.05 significance criterion. This pattern of results indicates that the map framed in light green predominated responses during the pretraining session (Fig. $4 c$, bar graphs). For the post-training, there was a significant interaction between stimulus lateralization and map $\left(F_{(1,9)}=9.77 ; p=0.012\right)$, whereas neither main effect reached our 0.05 significance criterion. Similar to the effects over the $195-250 \mathrm{~ms}$ interval, this pattern indicates that during the posttraining session, the map framed in gray predominated responses to the R500 lateralization, whereas the map framed in light green predominated responses to the R385 lateralization (Fig. 4c, bar graphs).

To complement the classification approach of the topographic pattern analysis and fitting procedure, we conducted a time-point by time-point statistical contrast of the global dissim- 


\section{Group-average LAURA source estimations (195-250ms)}
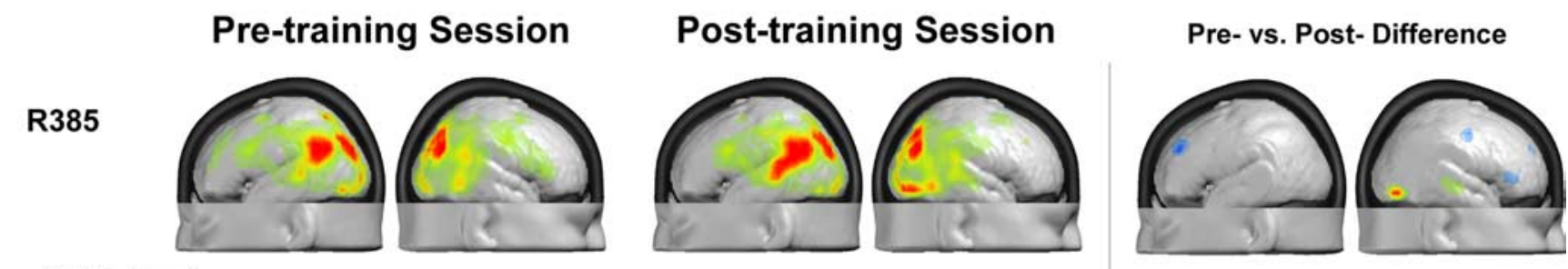

$3 \cdot 10^{-3} \mu \mathrm{A} / \mathrm{mm}^{3}$
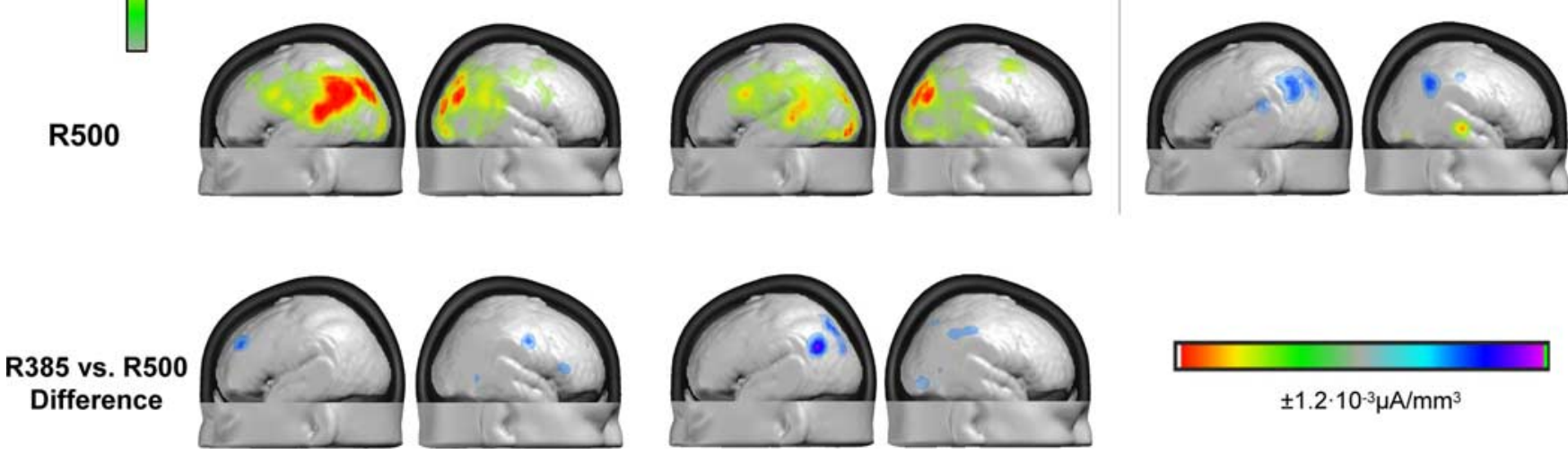

Figure 5. Source estimations. Group-averaged ( $n=10$ ) LAURA distributed linear source estimations were calculated over the $195-250 \mathrm{~ms}$ time period pretraining and post-training and are shown in response to the R385 and R500 conditions. The mean difference between these source estimations is also shown. Both the R385 and R500 lateralizations exhibited prominent sources within the parietal cortex bilaterally. Weaker sources were also observed in the prefrontal cortex. The difference revealed weaker activations to the R500 stimulus within the left inferior parietal cortex.

ilarity between responses to stimulus lateralizations of each session. The results of this analysis appear in Figure 3. This analysis confirmed that there was a significant topographic difference over the 195-215 $\mathrm{ms}$ and 271-307 ms intervals between responses to each lateralization during the post-training session. No significant differences were observed for the pretraining session of experiment 1 .

To assess both the generalization of these effects to other lateralizations and also to rule out an explanation of these results in terms of general repetition, we conducted an identical topographic pattern analysis and analyses of global dissimilarity on the AEPs from experiment 2. No time period showed a significant interaction between session, condition, and map (all $p$ values $>0.25$ ). Nor was there evidence of effects on global dissimilarity for either the pretraining or post-training session of experiment 2 .

\section{Source estimations}

The likely intracranial generators of these effects were visualized using the LAURA distributed linear inverse solution. Source estimations were calculated using the data from experiment 1 over the 195-250 ms interval (i.e., when topographic modulations were observed). Data from each subject were averaged across the 195-250 ms interval to generate to a single data point per subject for each session and lateralization, separately. Source estimations were then calculated and group averaged (Fig. 5). Both the R385 and R500 lateralizations exhibited prominent sources within the parietal cortex bilaterally. Weaker sources were also observed in the prefrontal cortex. The group-averaged difference between source estimations for these conditions (scaled at one-third the current density of the original source estimations) was also calculated for the pretraining and post-training sessions, separately.
This difference revealed weaker activations to the R500 stimulus within the left parietal cortex (maximal differences at $-50,-62$, $24 \mathrm{~mm}$ and $-18,-78,36 \mathrm{~mm}$ using the coordinate system of Talairach and Tournoux, 1988). These maxima correspond to Brodmann's areas 7 and 39, respectively.

\section{Discussion}

Auditory processing is thought to be mediated by partially segregated "what" and "where" pathways involved in sound recognition and localization, respectively (Rauschecker, 1998; Hackett et al., 1999; Clarke et al., 2000, 2002; Tian et al., 2001; Clarke and Thiran, 2004; De Santis et al., 2007). Previous studies have demonstrated plastic changes within the "what" stream as subjects learned to discriminate between frequency, duration, or intensity of sounds or between semantic features of complex environmental sounds or speech (Tremblay et al., 1998; Jäncke et al., 2001; Syka, 2002; Bergerbest et al., 2004; Gottselig et al., 2004). To our knowledge, ours is the first demonstration in humans that learning-induced plasticity is also observed for spatial "where" functions; the electrophysiological manifestations of which can be predicted by pretraining performance measures. In addition to providing insights on the neurophysiological mechanisms of auditory spatial representations, our results open possible training-based neurorehabilitation strategies (Bellmann et al., 2001; Spierer et al., 2007) involving repeated training sessions to induce long-lasting effects (Schindler et al., 2002).

Training for $40 \mathrm{~min}$ improved spatial discrimination performance and changed neurophysiological responses during subsequent passive listening. The time course of auditory perceptual learning is highly variable and depends on factors including the type of task and its difficulty as well as the complexity of the 
trained auditory stimuli (Watson, 1980). In our experiments, most subjects performed poorly during the first training block and showed significant improvements after completing the training. Such rapid modification of auditory spatial processing has been previously shown using similar stimuli (Wright and Fitzgerald, 2001) and has also been demonstrated by Recanzone (1998), in which visually driven alteration of auditory spatial representations occurs after a 20-30 min training period. Wright and Fitzgerald (2001) had subjects indicate whether a target sound location was presented in the first or second position in a pair of sounds lateralized with different ITDs or IIDs. Extending the training over days led to further improvement in discriminating stimuli lateralized with IID but not with ITDs. Similarly, experiment 3 provides no evidence for long-lasting training effects with ITD-defined lateralizations. Effects of training on AEPs recorded subsequently during passive listening were also specific to the more lateral and less frequently presented location (R500), indicative of a learning-induced MMN. Such a pattern of results is not readily explained by a general learning or practice effect. Before training, subjects were unable to discriminate reliably between the two spatial positions (R385 and R500), and there was no evidence of neural response differences. Rather, responses to both positions engaged indistinguishable parietotemporal networks bilaterally. Effects on AEPs were in terms of the electric field topography and by extension the configuration of the underlying brain generators. Source estimations localized these changes to the left inferior parietal cortices.

In addition to the post-training AEP effects being limited to one of the trained positions, there was no evidence of generalization of training-induced effects to either the symmetrical positions in the opposite hemispace (experiment 2) or to equidistant spatial separations either medially or laterally within the same hemispace (experiment 3 ). The extent of generalization is typically interpreted as indicative of the level of processing that is affected by training (Fahle, 2005). In animal models, learninginduced changes in neuronal tuning at relatively early processing stages are believed to reflect specific effects of training (Irvine et al., 2000). Several psychophysical studies in humans have demonstrated improvements in perceptual discrimination that were specific to the trained stimuli (Karni and Sagi, 1991; Ahissar and Hochstein, 1997; Hawkey et al., 2004; Fitzgerald and Wright, 2005) or stimulus feature (Wright et al., 2001). However, other studies reported some generalization to untrained stimuli [tone sequences and synthetic phonemes, in Gottselig et al. (2004) and Tremblay et al. (1997), respectively]. Effects in our study were restricted to the trained positions but did not depend on the specific order in which stimuli at these positions were presented or depend on whether this order matched that used during training. This pattern is consistent with mechanisms involving the refinement of spatial representations and/or coding for the trained locations, rather than the establishment of spatial anchors, mnemonic templates, or general improvement in discriminating ITD cues. The specificity of our effects suggests that training affected relatively low-level process. This is further supported by the fact that training modified AEP responses passively recorded after training.

Differences between neural responses to the two positions during the post-training recordings occurred over the 195-250 ms poststimulus interval. Two lines of evidence suggest that this period entails the encoding of cortical representations of auditory space, rather than higher order process. First, it corresponds to the time period over which the MMN response has been observed to changes in the position of a sequence of sounds, particularly when behavioral discrimination is difficult and/or when there is minimal separation in terms of the physical features of the stimuli (Näätänen and Escera, 2000). We interpret the response change during the MMN temporal window as indicating that traininginduced plasticity of the cortical representations of the two positions and/or the cortical mechanisms involved in position discrimination (Näätänen et al., 1978). It is worth noting that similarly early (and often earlier) effects have been observed on auditory processing (Woldorff and Hillyard, 1991), suggesting that the responses over the 195-250 ms period are not simply exogenous but rather also subject to endogenous processes. Second, the timing of the present effects are consistent with a multiphase model of auditory spatial processing proposed by our group (Ducommun et al., 2002; Tardif et al., 2006), wherein different spatial cues are integrated over the $\sim 140-255 \mathrm{~ms}$ period and wherein spatial information is consolidated within different reference frames over the $\sim 255-400$ ms period.

The electrical neuroimaging analyses conducted in this study permit some speculation on the likely mechanism mediating training-induced plasticity of auditory spatial representations, beyond simply invoking the emergence of the MMN. Several types of neurophysiological modifications are conceivable, including alterations in the quantity of neurons recruited to respond to stimulation of a particular spatial location, in the synchrony of such neural responses, and in the spatial tuning of neural populations (for review, see Gilbert et al., 2001; Ohl and Scheich, 2005). Effects in our study were limited to topographic changes in the response to the more lateral and less frequently presented of the two simulated positions, with no evidence of modifications in response strength, indicative of generator changes that result in an MMN. Such results run counter to what would have been predicted had either neuronal recruitment or response synchrony been modified. For one, these mechanisms would likely not have specifically affected just one of the stimulated positions. Likewise, responses to both positions would be affected in the case of a general attention or arousal mechanism or a general learning-induced change in spatial coding. Second, these mechanisms would likely have resulted in a change in the strength of responses, rather than the configuration of underlying brain generators.

Our results are consistent with the refinement of neuronal spatial tuning at a population level. Previous studies have shown that pitch training was accompanied by an increase in neuronal selectivity and decrease of the corresponding cortical representation (Weinberger et al., 1990; Edeline et al., 1993; Recanzone et al., 1993). The present study revealed that training selectively changed the topography of the electric field yielded by the target stimulus by decreasing the activity of sources within left inferior parietal cortices (Fig. 5). A putative neural mechanism may involve inhibitory processes in generating plasticity via the exclusion of the activity of less specific neurons (Ghose, 2004; Ohl and Scheich, 2005) or noise-related responses (Rainer et al., 2004). Others have further extended this notion in terms of refining a perceptual template (Li et al., 2004), wherein those neurons that respond most strongly might not actually convey the greatest amount of information regarding a learned discrimination. Rather, greater differential responses to the spatial positions may instead occur in neurons exhibiting weaker response magnitude. In which case, it would be beneficial to inhibit such strongly responding neurons to produce a more informative response profile (Ghose, 2004). On the whole, our results lend additional support to the concept of auditory spatial representations based on population coding, which have been proposed in animal mod- 
els (Recanzone et al., 1993; Rauschecker and Tian, 2000; King et al., 2001; Middlebrooks et al., 2002; Woods et al., 2006).

Source estimations localized the present effects to regions of the left inferior parietal cortex (Fig. 5). Parietal and posterior temporal cortices have been shown to be implicated in auditory spatial functions in man (Griffiths et al., 1998; Bushara et al., 1999; Maeder et al., 2001; Weeks et al., 2001; Ducommun et al., 2002; Deouell et al., 2006; Sonnadara et al., 2006; Tardif et al., 2006; De Santis et al., 2007) and nonhuman primate (Stricane et al., 1996; Schlack et al., 2005). Previous studies on ITD processing have shown that left hemisphere responds more specifically to stimuli lateralized in the right hemispace whereas right hemisphere respond to stimuli in the left or right hemispace (Kuwada and Yin, 1983; Caird and Klinke, 1987).

\section{References}

Ahissar M, Hochstein S (1997) Task difficulty and the specificity of perceptual learning. Nature 387:401-406.

Altman YA, Vaitulevich SF, Shestopalova LB (2004) Changes in evoked potentials during the action of sound signals with different localizing characteristics. Neurosci Behav Physiol 34:139-146.

Atienza M, Cantero JL, Dominguez-Marin E (2002) The time course of neural changes underlying auditory perceptual learning. Learn Mem 9:138-150.

Atienza M, Cantero JL, Quian Quiroga R (2005) Precise timing accounts for post-training sleep-dependent enhancements of the auditory mismatch negativity. NeuroImage 26:628-634.

Barbay S, Peden EK, Falchook G, Nudo RJ (1999) Sensitivity of neurons in somatosensory cortex (S1) to cutaneous stimulation of the hindlimb immediately following a sciatic nerve crush. Somatosens Mot Res 16:103-114.

Bellmann A, Meuli R, Clarke S (2001) Two types of auditory neglect. Brain 124:676-687.

Bergerbest D, Ghahremani DG, Gabrieli JD (2004) Neural correlates of auditory repetition priming: reduced fMRI activation in the auditory cortex. J Cogn Neurosci 16:966-977.

Blauert J (1997) Spatial Hearing (revised edition). Cambridge, MA: MIT.

Brandeis D, Lehmann D, Michel CM, Mingrone W (1995) Mapping eventrelated brain potential microstates to sentence endings. Brain Topogr 8:145-159.

Bushara KO, Weeks RA, Ishii K, Catalan MJ, Tian B, Rauschecker JP, Hallett M (1999) Modality-specific frontal and parietal areas for auditory and visual spatial localization in humans. Nat Neurosci 2:759-766.

Caird DM, Klinke R (1987) Processing of interaural time and intensity differences in the cat inferior colliculus. Exp Brain Res 68:379-392.

Calford MB, Tweedale R (1988) Immediate and chronic changes in responses of somatosensory cortex in adult flying-fox after digit amputation. Nature 332:446-448.

Calford MB, Wright LL, Metha AB, Taglianetti V (2003) Topographic plasticity in primary visual cortex is mediated by local corticocortical connections. J Neurosci 23:6434-6442.

Clarke S, Thiran AB (2004) Auditory neglect: what and where in auditory space. Cortex 40:291-300.

Clarke S, Bellmann A, Meuli RA, Assal G, Steck AJ (2000) Auditory agnosia and auditory spatial deficits following left hemispheric lesions: evidence for distinct processing pathways. Neuropsychologia 38:797-807.

Clarke S, Bellmann A, Thiran A, Maeder P, Adriani M, Vernet O, Regli L, Cuisenaire O, Thiran JP (2002) What and where in human audition: selective deficits following focal hemispheric lesions. Exp Brain Res 147:8-15.

Deouell LY, Parnes A, Pickard N, Knight RT (2006) Spatial location is accurately tracked by human auditory sensory memory: evidence from the mismatch negativity. Eur J Neurosci 24:1488-1494.

Dinse HR, Godde B, Hilger T, Haupt SS, Spengler F, Zepka R (1997) Shortterm functional plasticity of cortical and thalamic sensory representations and its implication for information processing. Adv Neurol 73:159-178.

De Santis L, Clarke S, Murray MM (2007) Automatic and intrinsic auditory "what" and "where" processing in humans revealed by electrical neuroimaging. Cereb Cortex 17:9-17.
Doetsch GS, Harrison TA, MacDonald AC, Litaker MS (1996) Short-term plasticity in primary somatosensory cortex of the rat: rapid changes in magnitudes and latencies of neuronal responses following digit denervation. Exp Brain Res 112:505-512.

Donoghue JP, Suner S, Sanes JN (1990) Dynamic organization of primary motor cortex output to target muscles in adult rats. II. Rapid reorganization following motor nerve lesions. Exp Brain Res 79:492-503.

Ducommun CY, Murray MM, Thut G, Bellmann A, Viaud-Delmon I, Clarke S, Michel CM (2002) Segregated processing of auditory motion and auditory location: an ERP mapping study. NeuroImage 16:76-88.

Edeline JM, Pham P, Weinberger NM (1993) Rapid development of learning-induced receptive field plasticity in the auditory cortex. Behav Neurosci 107:539-551.

Fahle M (2005) Perceptual learning: specificity versus generalization. Curr Opin Neurobiol 15:154-160.

Faggin BM, Nguyen KT, Nicolelis MA (1997) Immediate and simultaneous sensory reorganization at cortical and subcortical levels of the somatosensory system. Proc Natl Acad Sci USA 94:9428-9433.

Fitzgerald MB, Wright BA (2005) A perceptual learning investigation of the pitch elicited by amplitude-modulated noise. J Acoust Soc Am 118:3794-3803.

Fritz J, Shamma S, Elhilali M, Klein D (2003) Rapid task-related plasticity of spectrotemporal receptive fields in primary auditory cortex. Nat Neurosci 6:1216-1223.

Fritz J, Elhilali M, Shamma S (2005) Active listening: task-dependent plasticity of spectrotemporal receptive fields in primary auditory cortex. Hear Res 206:159-176.

Garraghty PE, Kaas JH (1991) Functional reorganization in adult monkey thalamus after peripheral nerve injury. NeuroReport 2:747-750.

Ghose GM (2004) Learning in mammalian sensory cortex. Curr Opin Neurobiol 14:513-518.

Gilbert CD, Wiesel TN (1992) Receptive field dynamics in adult primary visual cortex. Nature 356:150-152.

Gilbert CD, Sigman M, Crist RE (2001) The neural basis of perceptual learning. Neuron 31:681-697.

Gottselig JM, Brandeis D, Hofer-Tinguely G, Borbely AA, Achermann P (2004) Human central auditory plasticity associated with tone sequence learning. Learn Mem 11:162-171.

Grave de Peralta Menendez R, Gonzalez Andino S, Lantz G, Michel CM, Landis T (2001) Noninvasive localization of electromagnetic epileptic activity. I. Method descriptions and simulations. Brain Topogr 14:131-137.

Grave de Peralta Menendez R, Murray MM, Michel CM, Martuzzi R, Gonzalez Andino SL (2004) Electrical neuroimaging based on biophysical constraints. NeuroImage 21:527-539.

Green D, Swets J (1966) Signal detection: theory and psychophysics. New York: Wiley.

Griffiths TD, Buchel C, Frackowiak RS, Patterson RD (1998) Analysis of temporal structure in sound by the human brain. Nat Neurosci $1: 422-427$

Guthrie D, Buchwald JS (1991) Significance testing of difference potentials. Psychophysiology 28:240-244.

Hackett TA, Stepniewska I, Kaas JH (1999) Prefrontal connections of the parabelt auditory cortex in macaque monkeys. Brain Res 817:45-58.

Hawkey DJ, Amitay S, Moore DR (2004) Early and rapid perceptual learning. Nat Neurosci 7:1055-1056.

Huntley GW (1997) Correlation between patterns of horizontal connectivity and the extent of short-term representational plasticity in rat motor cortex. Cereb Cortex 7:143-156.

Irvine DR, Martin RL, Klimkeit E, Smith R (2000) Specificity of perceptual learning in a frequency discrimination task. J Acoust Soc Am 108:2964-2968.

Iriki A, Tanaka M, Iwamura Y (1996) Coding of modified body schema during tool use by macaque postcentral neurones. NeuroReport 7:2325-2330.

Iwamura Y (2000) Bilateral receptive field neurons and callosal connections in the somatosensory cortex. Philos Trans R Soc Lond B Biol Sci 355:267-273.

Jäncke L, Gaab N, Wustenberg T, Scheich H, Heinze HJ (2001) Short-term functional plasticity in the human auditory cortex: an fMRI study. Brain Res Cogn Brain Res 12:479-485.

Karni A, Sagi D (1991) Where practice makes perfect in texture discrimina- 
tion: evidence for primary visual cortex plasticity. Proc Natl Acad Sci USA 88:4966-4970.

King AJ (2006) Auditory neuroscience: activating the cortex without sound. Curr Biol 2006 16:410-411.

King AJ, Schnupp JWH, Doubell TP (2001) The shape of ears to come: dynamic coding of auditory space. Trends Cogn Sci 5:261-270.

Kraus N, McGee T, Carrell TD, King C, Tremblay K, Nicol T (1995) Central auditory system plasticity associated with speech discrimination training. J Cogn Neurosci 7:25-32.

Krzanowski W, Lai YT (1985) A criterion for determining the number of groups in a data set using sum of squares clustering. Biometrics 44:23-34.

Kuwada S, Yin TC (1983) Binaural interaction in low-frequency neurons in inferior colliculus of the cat. I. Effects of long interaural delays, intensity, and repetition rate on interaural delay function. J Neurophysiol 50:981-999.

Lehmann D (1987) Principles of spatial analysis. In: Handbook of electroencephalography and clinical neurophysiology. In: Methods of analysis of brain electrical and magnetic signals, Vol I (Gevins AS, Remond A, eds), pp 309-354. Amsterdam: Elsevier.

Lehmann D, Skrandies W (1980) Reference-free identification of components of checkerboard-evoked multichannel potential fields. Electroencephalogr Clin Neurophysiol 48:609-621.

Li RW, Levi DM, Klein SA (2004) Perceptual learning improves efficiency by re-tuning the decision 'template' for position discrimination. Nat Neurosci 7:178-183.

Macmillan N, Creelman C (1991) Detection theory: a user's guide. UK: Cambridge UP.

Maeder PP, Meuli RA, Adriani M, Bellmann A, Fornari E, Thiran JP, Pittet A, Clarke S (2001) Distinct pathways involved in sound recognition and localization: a human fMRI study. NeuroImage 14:802-816.

Manly BF (1991) Randomization and Monte Carlo methods in biology. London: Chapman and Hall.

Menning H, Roberts LE, Pantev C (2000) Plastic changes in the auditory cortex induced by intensive frequency discrimination training. NeuroReport 11:817-822.

Michel CM, Murray MM, Lantz G, Gonzales S, Spinelli L, Grave de Peralta R (2004) EEG source imaging. Clin Neurophysiol 115:2195-2222.

Middlebrooks JC, Xu L, Furukawa S, Macpherson EA (2002) Cortical neurons that localize sounds. Neuroscientist 8:73-83.

Murray MM, Michel CM, Grave de Peralta R, Ortigue S, Brunet D, Gonzales Andino S, Schnider A (2004) Rapid discrimination of visual and multisensory memories revealed by electrical neuroimaging. NeuroImage 21:125-135.

Murray MM, Imber ML, Javitt DC, Foxe JJ (2006) Boundary completion is automatic and distinct from shape discrimination. J Neurosci 26: 12043-12054.

Näätänen R, Escera C (2000) Mismatch negativity: clinical and other applications. Audiol Neurootol 5:105-110.

Näätänen R, Gaillard AWK, Mäntysalo S (1978) Early selective attention effect on evoked potential reinterpreted. Acta Psychol 42:313-329.

Näätänen R, Schröger E, Karakas S, Tervaniemi M, Paavilainen P (1993) Development of a memory trace for a complex sound in the human brain. NeuroReport 4:503-506.

Nager W, Kohlmetz C, Joppich G, Mobes J, Munte TF (2003) Tracking of multiple sound sources defined by interaural time differences: brain potential evidence in humans. Neurosci Lett 344:181-184.

Ohl FW, Scheich H (2005) Learning-induced plasticity in animal and human auditory cortex. Curr Opin Neurobiol 15:470-477.

Oldfield RC (1971) The assessment and analysis of handedness: the Edinburgh inventory. Neuropsychologia 9:97-113.

Paavilainen P, Karlsson ML, Reinikainen K, Näätänen R (1989) Mismatch negativity to change in spatial location of an auditory stimulus. Electroencephalogr Clin Neurophysiol 73:129-141.

Perrin F, Pernier J, Bertrand O, Girad MH, Echalier JF (1987) Mapping of scalp potentials by surface spline interpolation. Electroencephalogr Clin Neurophysiol 72:184-189.
Rainer G, Lee H, Logothetis NK (2004) The effect of learning on the function of monkey extrastriate visual cortex. PLoS Biol 2:E44.

Rauschecker JP (1998) Cortical processing of complex sounds. Curr Opin Neurobiol 8:516-521.

Rauschecker JP, Tian B (2000) Mechanisms and streams for processing of "what" and "where" in auditory cortex. Proc Natl Acad Sci USA 97:11800-11806.

Recanzone GH (1998) Rapidly induced auditory plasticity: the ventriloquism aftereffect. Proc Natl Acad Sci USA 95:869-875.

Recanzone GH, Schreiner CE, Merzenich MM (1993) Plasticity in the frequency representation of primary auditory cortex following discrimination training in adult owl monkeys. J Neurosci 13:87-103.

Schindler I, Kerkhoff G, Karnath HO, Keller I, Goldenberg G (2002) Neck muscle vibration induces lasting recovery in spatial neglect. J Neurol Neurosurg Psychiatry 73:412-419.

Schlack A, Sterbing-D’Angelo SJ, Hartung K, Hoffmann KP, Bremmer F (2005) Multisensory space representations in the macaque ventral intraparietal area. J Neurosci 25:4616-4625.

Schröger E, Wolff C (1996) Mismatch response of the human brain to changes in sound location. NeuroReport 7:3005-3008.

Sonnadara RR, Alain C, Trainor LJ (2006) Effects of spatial separation and stimulus probability on the event-related potentials elicited by occasional changes in sound location. Brain Res 1071:175-185.

Spierer L, Meuli R, Clarke S (2007) Extinction of auditory stimuli in hemineglect: space versus ear. Neuropsychologia 45:540-551.

Stricane B, Andersen RA, Mazzoni P (1996) Eye-centered, head-centered, and intermediate coding of remembered sound locations in area LIP. J Neurophysiol 76:2071-2076.

Sur M, Leamey CA (2001) Development and plasticity of cortical areas and networks. Nat Rev Neurosci 2:251-262.

Syka J (2002) Plastic changes in the central auditory system after hearing loss, restoration of function, and during learning. Physiol Rev 82:601-636.

Talairach J, Tournoux P (1988) Co-planar stereotaxic atlas of the human brain. New York: Thieme.

Tardif E, Murray MM, Meylan R, Spierer L, Clarke S (2006) The spatiotemporal brain dynamics of processing and integrating sound localization cues in humans. Brain Res 1092:161-176.

Tian B, Resa D, Durham A, Kustov A, Rauschecker JP (2001) Function of specialization in rhesus monkey auditory cortex. Science 292:290-293.

Tibshirani R, Walther G, Botstein D, Brown P (2005) Cluster validation by prediction strength. J Comput Graphical Stat 14:511-528.

Tremblay K, Kraus N, Carrell TD, McGee T (1997) Central auditory system plasticity: generalization to novel stimuli following listening training. J Acous Soc Am 102:3762-3773.

Tremblay K, Kraus N, McGee T (1998) The time course of auditory perceptual learning: neurophysiological changes during speech-sound training. NeuroReport 9:3557-3560.

Watson CS (1980) Time course of auditory perceptual learning. Ann Otol Rhinol Laryngol Suppl 89:96-102.

Weeks RA, Aziz-Sultan A, Bushara KO, Tian B, Wessinger CM, Dang N, Rauschecker JP, Hallett M (2001) A PET study of human auditory spatial processing. Neurosci Lett 262:155-158.

Weinberger NM, Ashe JH, Metherate R, McKenna TM, Diamond DM, Bakin J (1990) Returning auditory cortex by learning: a preliminary model of receptive field plasticity. Concepts Neurosci 1:9 1-1 32.

Woldorff MG, Hillyard SA (1991) Modulation of early auditory processing during selective listening to rapidly presented tones. Electroencephalogr Clin Neurophysiol 79:170-191.

Woods TM, Lopez SE, Long JH, Rahman JE, Recanzone GH (2006) Effects of stimulus azimuth and intensity on the single neuron activity in the auditory cortex of the alert macaque monkey. J Neurophysiol 96:3323-3337.

Wright BA, Fitzgerald MB (2001) Different patterns of human discrimination learning for two interaural cues to sound-source location. Proc Natl Acad Sci USA 98:12307-12312. 